\section{DUAL BLOCKADE OF LAG3 AND TIGIT IMPROVES THE TREATMENT EFFICACY OF A NANOPARTICLE-MEDIATED IMMUNORADIATION IN ANTI-PD1 RESISTANT LUNG CANCER IN MICE}

${ }^{1}$ Yun $\mathrm{Hu}^{*}$, ${ }^{1}$ James Welsh, ${ }^{2}$ Sebastien Paris, ${ }^{1}$ Genevieve Bertolet, ${ }^{1}$ Hampartsoum Barsoumian, 'Lily Schuda, 'Kewen He, 'Duygu Sezen, 'Mark Wasley, ${ }^{1}$ Joylise Mitchell, ${ }^{1}$ Tiffany Voss, ${ }^{1}$ Fatemeh Masrorpour, ${ }^{2}$ SILVA Jordan, ${ }^{1}$ Claudia Kettlun Leyton, 'Liangpeng Yang, 'Nahum Puebla-Osorio, 'Saumil Gandhi, 'QuynhNhu Nguyen, 'Angelica Cortez. 'MD Anderson Cancer Center, Houston, TX, USA; ${ }^{2}$ Nanobiotix, Paris, France

Background TIGIT and LAG3 are inhibitory receptors expressed on cytotoxic CD8 $+\mathrm{T}$ cells and NK cells and directly inhibit the activation and proliferation of these cells. We proposed that blockade of TIGIT and LAG3 could improve antitumor immune response in a mouse model of anti-PD1 (aPD1)-resistant mice.

Methods 129Sv/Ev mice were inoculated with 50,000 aPD1resistant 344SQR cells in the right leg on day 0 (primary tumor) and with 50,000 cells in the left leg on day 4 (secondary tumor). Primary tumors were injected with NBTXR3 radioenhancer nanoparticles on day 7 and irradiated with 12 Gy on days 8, 9, and 10. Anti-PD1, aLAG3, and aTIGIT were given to mice by intraperitoneal injections on days 5,8 , $11,14,21,28,35$, and 42 . On day 21 , primary tumors, secondary tumors, and blood samples were harvested and analyzed with flow cytometry to evaluate changes in immune cell populations. The RNA extracted from the tumors were also analyzed by Nanostring. Mice in which tumors were completely eradicated were re-challenged with another 50,000 344SQR cells in the right flank at least two months post radiation; no further treatment was given to these mice, and tumor growth was monitored.

Results The addition of aTIGIT, aLAG3, or aTIGIT+aLAG3 to NBTXR3+XRT+aPD1 therapy significantly improved control of tumors, and the addition of aTIGIT +aLAG3 also led to fewer spontaneous lung metastases. The addition of either aTIGIT or aLAG3 to NBTXR3+XRT+aPD1 extended mouse survival time relative to NBTXR3+XRT + aPD1. None of the 8 mice in either the NBTXR3 + XRT +aPD1+aTIGIT group or the NBTXR3+XRT+aPD1+aLAG3 group survived more than 32 days; in contrast, 3 of the 8 mice that received NBTXR3 $+\mathrm{XRT}+\mathrm{aPD} 1+\mathrm{aTIGIT}+\mathrm{aLAG} 3$ survived until the end of the experiment. These surviving mice were found to have developed memory against 344SQR cells, and no further tumor growth was observed after re-challenge. Flow cytometry analysis showed that adding aTIGIT+aLAG3 to NBTXR3+XRT + aPD1 increased the percentages of proliferating CD8 $+\mathrm{T}$ cells in primary tumors, secondary tumors, and blood. Furthermore, Nanostring transcriptomic analysis of cells isolated from the tumors of mice thus treated showed evidence of classical two-step immunological priming, with an elevation of innate immune genes at the primary tumor and full-blown activation of the immune system within the secondary tumor.

Conclusions Blockade of TIGIT and LAG3 with NBTXR3 +XRT+aPD1 improved CD8 + T-cell proliferation, augmented the antitumor response at both irradiated and unirradiated (abscopal) tumors, and induced potent long-term antitumor memory in mice.

Acknowledgements This work was supported by Cancer Center Support (Core) Grant CA016672 to The University of Texas MD Anderson Cancer Center; the Goodwin family research fund; the family of $\mathrm{M}$. Adnan Hamed and the Orr Family Foundation to MD Anderson Cancer Center's Thoracic
Radiation Oncology program; an MD Anderson Knowledge Gap award; Nanobiotix.

http://dx.doi.org/10.1136/jitc-2021-SITC2021.575 